\title{
Stability-considered Density-adaptive Routing Protocol with Fuzzy Logic Control Optimum Angle in Mobile Wireless Sensor Networks
}

\author{
Young-Long Chen, Yu-Ling Zeng, Hsin-Yu Chen, and Yan-Hao Lin
}

Department of Computer Science and Information Engineering,

National Taichung University of Science and Technology, No. 129, Sec. 3, Sanmin Rd., Taichung 404, Taiwan

(Received March 28, 2018; accepted September 7, 2018)

Keywords: stability, density, angle, fuzzy logic control

To reduce the energy consumption of the routing protocol, we propose a stability-considered density-adaptive routing protocol with fuzzy logic control (SDRFLC) to improve the stabilityconsidered density-adaptive routing protocol (SDR). In the initial stage, the proposed method determines whether a relay node is needed in accordance with the distance between source and destination nodes. If the relay node is required, then density is calculated. On the basis of this density, the best routing mode is selected. In the newest sparse mode (S-mode), the SDRFLC uses the stability and density values as the input of fuzzy logic control. Then, the output value is used to adjust the angle and solve the local maximum problem. Our proposed method is superior to the SDR in terms of the transmission ratio and routing overhead. With higher stability, our proposed method can effectively control the route requirement of sensor nodes (SNs) and prolong the life of mobile wireless sensor networks (MWSNs).

\section{Introduction}

In recent years, mobile wireless sensor networks $(\mathrm{MWSNs})^{(1,2)}$ have received considerable research attention. Since MWSNs are non-infrastructural, it is not necessary to build the basic environment in advance. ${ }^{(3)}$ In a MWSN, mobile sensor nodes (SNs) not only transmit data between each other, but also act as routers. Because of the SN mobility, connections between SNs are uncertain, resulting in unstable data transmission ${ }^{(4)}$ and increased energy consumption, thereby reducing SN lifespans. Many studies have therefore focused on extending wireless network lifespans ${ }^{(5)}$ using particular routing algorithms ${ }^{(6)}$ and transmission paths..$^{(7)}$

One of the most important issues of MWSN development is energy consumption. Many studies concern routing, communication, and quality of service (QoS) applications ${ }^{(8)}$ in MWSNs. Since MWSNs SNs are movable, the network topology is changeable during the network lifetime. As a result, $\operatorname{QoS}^{(9)}$ is unable to guarantee reliable transmission, and the network may experience packet loss, delay, jitter, low throughput, and delivery problems. In light of this situation, many studies have proposed enhanced routing protocols, including the optimized link state routing protocol (OLSR). ${ }^{(10)}$ OLSR is able to reduce message overhead by

*Corresponding author: e-mail: ylchen66@nutc.edu.tw https://doi.org/10.18494/SAM.2018.2050 
using multipoint relays to forward broadcast messages by flooding, but requires large, dense networks. Haas proposed the zone routing protocol (ZRP) ${ }^{(11)}$ which is a hybrid routing protocol with proactive and reactive routing schemes. It is able to accelerate delivery and reduce control messages. Karp and Kung proposed greedy perimeter stateless routing (GPSR), ${ }^{(12)}$ which has become a common routing protocol. Geographic routing uses greedy forwarding to find the shortest distance between SNs in order to make forwarding decisions. GPSR addresses the problem of greedy forwarding regions by routing around the perimeter of these regions.

For optimal routing path selection, Arora and Monga proposed the ad hoc on-demand distance vector (AODV) ${ }^{(13)}$ with a knowledge-based algorithm. It establishes the optimal routing path using the AODV and improves the link failure using the knowledge-based learning algorithm. The fuzzy-based geographic forwarding protocol (FuGeF) ${ }^{(14)}$ was proposed to mitigate spatiotemporal attack and provide minimal security. FuGeF introduces three forwarding node selection parameters of remaining energy, cost of connection, and forwarding distance selection via the fuzzy logic system (FLS) with a novel form of dynamism. The stability-considered density-adaptive routing protocol $(\mathrm{SDR})^{(15)}$ is a novel MWSN routing protocol that is able to reduce the rebuild of routes and guarantee stability with two routing mechanisms: dense mode (D-mode) and sparse mode (S-mode).

To reduce energy consumption by means of a routing protocol, we propose a stabilityconsidered density-adaptive routing protocol with fuzzy logic control (SDRFLC) to improve the SDR. In the initial stage, the proposed method yields the distance from the source node to the destination node. Then, the optimum angle is used in the density calculation mechanism. The best routing mode will be selected in accordance with the density. In the newest S-mode, the SDRFLC uses the stability and density values with fuzzy logic control ${ }^{(16,17)}$ to address the local maximum problem.

\section{Materials and Methods}

The routing processes of the SDRFLC are based on the SDR, as depicted in Fig. 1. First, in the initial range decision, the transmission distance $d$ from the source to the destination is compared with the threshold. If the distance is smaller than the threshold, the density calculation mechanism is used to calculate the stability and density of the optimum destination face area (DFA). The corresponding routing mode will be selected in accordance with the density. When the density is higher than the threshold, the SDRFLC will use the original D-mode to forward the data. If the density is lower, the SDRFLC will use the new S-mode $\left(\mathrm{S}^{+}\right.$-mode) to find the forwarding node. The $\mathrm{S}^{+}$-mode utilizes fuzzy logic control to increase the search angle. If this search angle still does not lead to the forwarding node, the node will use the stability-based face routing mechanism to find the forwarding node.

\subsection{Initial range decision}

The first-order radio model (FORM) energy model ${ }^{(18)}$ is used to obtain the distance threshold $d_{\text {threshold }}$ to determine whether the forwarding node is required to transfer data. In 


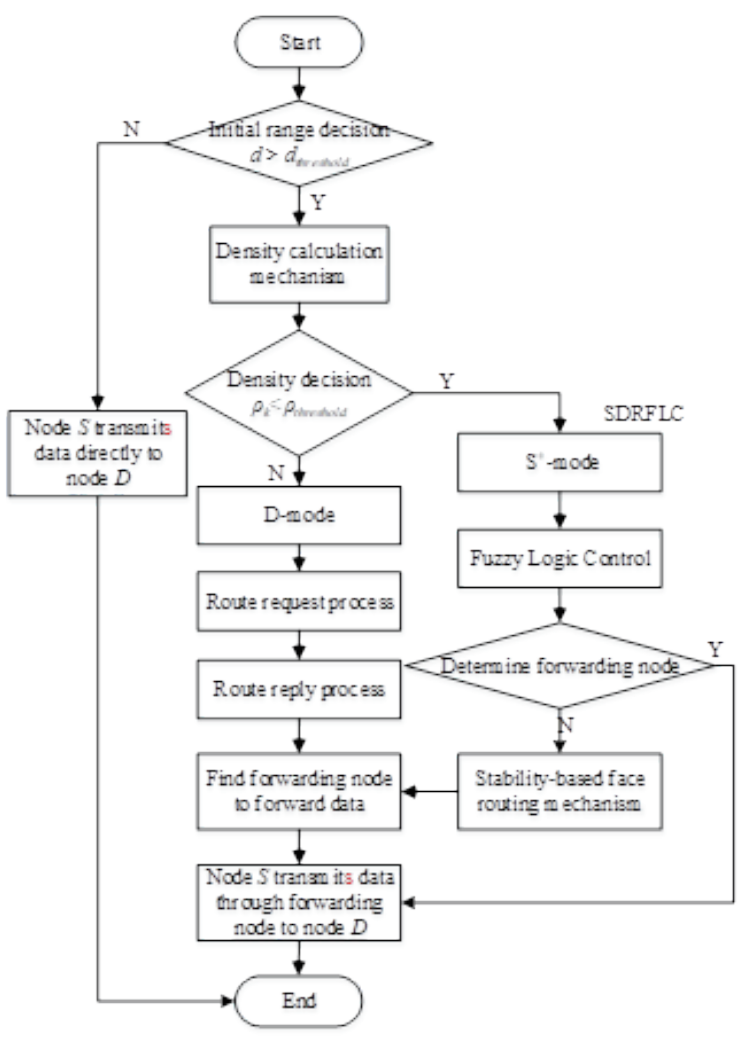

Fig. 1. Flow of SDRFLC.

the FORM, a data packet of $n$ bits is assumed. $E_{\text {total }}$ is the total energy consumption to transmit from source node $S$ to destination node $D$. If $d>d_{\text {threshold }}$, the source transfers the data directly; if $d>d_{\text {threshold }}$, source node $S$ needs a forwarding node to overcome the transmission range problem.

$$
\begin{aligned}
& \begin{aligned}
E_{\text {total }} & =E_{T x}+E_{R x} \\
& =n\left(E_{\text {elec }}+\varepsilon_{\text {amp }} \times d^{\alpha}\right)+n \times E_{\text {elec }} \\
& =n\left(2 E_{\text {elec }}+\varepsilon_{\text {amp }} \times d^{\alpha}\right)
\end{aligned} \\
& d_{\text {threshold }}=\alpha \sqrt{2 E_{\text {elec }} / \varepsilon_{\text {amp }}\left(1-2^{1-\alpha}\right)}
\end{aligned}
$$

\subsection{Density calculation mechanism for routing mode selection}

In the density calculation mechanism, SDR-Angle calculates the search radius of S-D, only 90 degrees, as DFA, which brings the forwarding node closer to the S-D datum line. Then, the density calculation mechanism calculates the node density in the DFA. 


\subsubsection{Evaluation of DFA stability}

Consider a MWSN described by $G(t)=(V, E(t))$, where nodes are denoted by $V=\{1,2, \ldots, i\}$. The sets of routing paths are denoted by $E(t)=\left\{E_{1}, E_{2}, \ldots, E_{j}\right\}$. If node $l$ receives a message from node $k$, it shows that nodes $l$ and $k$ exist at a directed edge, $e(l, k) . E_{l}\left(t_{k}\right)$ and $E_{l}\left(t_{k+1}\right)$ show that node $l$ and its neighbor node form a path at two adjacent times. The stability $S_{l}$ can be calculated as

$$
S_{l}=\frac{1}{l-1} \sum_{k=1}^{l-1} \cos \theta_{k}=\frac{1}{l-1} \sum_{k=1}^{l-1} \frac{E_{l}\left(t_{k}\right) E_{l}\left(t_{k+1}\right)}{\left|E_{l}\left(t_{k}\right) \| E_{l}\left(t_{k+1}\right)\right|}
$$

As shown in Eq. (3), when the stability $S_{l}$ is large, the angle $\theta_{k}$ of $E_{l}\left(t_{k}\right)$ to $E_{l}\left(t_{k+1}\right)$ will be small, which means that the nodes do not change dynamically in adjacent times, and node $i$ is relatively stable. If the stability $S_{l}$ is small, the angle $\theta_{k}$ of $E_{l}\left(t_{k}\right)$ to $E_{l}\left(t_{k+1}\right)$ will be large, which means that the nodes change dynamically in adjacent times, and node $l$ moves rapidly.

\subsubsection{DFA and stability considered in density assessment}

Node S searches within a broadcast angle of 90 degrees to discover existing nodes in its adjacent area. In the density assessment, the density $\rho_{k}$ of the search area in the DFA is calculated as

$$
\rho_{k}=\frac{\sum_{i \in D F A_{90}} S_{l}}{D F A_{90}}=\frac{4 \sum_{i \in D F A_{90}} S_{l}}{\pi r^{2} / 2}
$$

where $D F A_{90}$ is the search area of the DFA. $\rho_{k}$ is the nearby node density of node $k$ and $r$ is the transmission range of neighbor node $k$.

The corresponding routing mode is selected on the basis of the density $\rho_{k}$. When $\rho_{k}>\rho_{\text {threshold}}$, the basic D-mode will be triggered. When $\rho_{k}>\rho_{\text {threshold }}$, the $\mathrm{S}^{+}$-mode will be triggered.

\section{$2.3 \mathrm{~S}^{+}$-mode}

If the density $\rho_{k}>\rho_{\text {threshold, }}$, it expresses sparse forwarding nodes in the area. The $\mathrm{S}^{+}$-mode will be described in detail below.

\subsubsection{Local maximum problem}

If node $S$ transmits to node $D$, there is only one path and the neighbor nodes are not in the DFA search area. This problem is called the local maximum problem. 
The situation is such that the final node is unable to find another node by which to transfer data to destination node $D$ in the local maximum problem. The final node is not the destination node and is thus called a dead-end or concave node. As shown in Fig. 2, when the $\rho_{k}$ of the dead-end node is smaller than the threshold, the routing mode should switch to the $\mathrm{S}^{+}$-mode to solve the local maximum problem. Therefore, in this study, the stability-based face routing mechanism is used to address this problem.

\subsubsection{Enlargement of DFA angle in fuzzy logic control}

When the current node becomes a dead-end node, the proposed method utilizes fuzzy logic control to obtain a new search angle. As shown in Fig. 3, first, the density and stability are fuzzified. The values are then given corresponding fuzzy variables with fuzzy rules and fuzzy inference. Because there are two inputs with three varieties, nine rules are obtained for the system, as shown in Table 1.

The angle result will be used to find a forwarding node in the $\mathrm{S}^{+}$-mode. If the enlarged angle still cannot find forwarding nodes, the stability-based face routing mechanism will be used to find a forwarding node.

\subsubsection{Stability-based face routing mechanism}

The $\mathrm{S}^{+}$-mode is able to deal with the local maximum problem because the stabilityconsidered Gabriel graph algorithm (SGG) considers the stability of linked nodes. As shown in Fig. 4 , it is assumed that the stability $S_{a}$ of node a is smaller than $S_{\text {threshold }}$. When nodes $b$ and

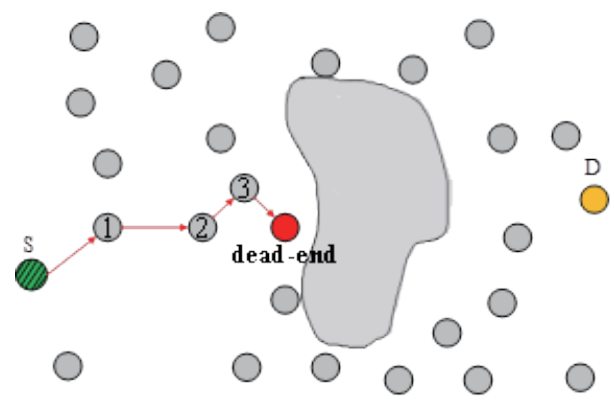

Fig. 2. (Color online) Local maximum problem.

Table 1

Angle of fuzzy rules.

\begin{tabular}{lccc}
\hline \multirow{2}{*}{ Stability } & \multicolumn{3}{c}{ Density } \\
\cline { 2 - 4 } & High & Medium & Low \\
\hline High & Low & Low & High \\
Medium & Low & Medium & High \\
Low & Low & Medium & High \\
\hline
\end{tabular}

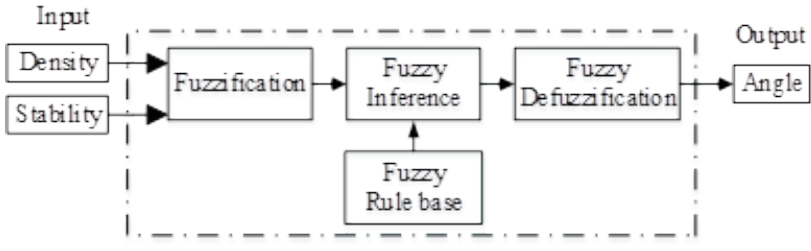

Fig. 3. Fuzzy logic control model.

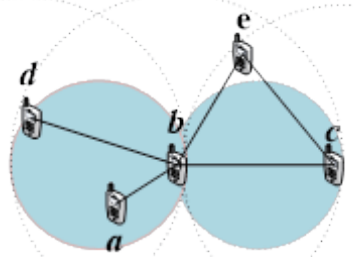

Fig. 4. (Color online) SGG schematic. 
$d$ build a link, node $a$ is not considered because link $b-d$ can transfer data in the transmission range, and the stability $S_{a}$ of node $a$ is smaller than the threshold. As another example, it is assumed that the stability $S_{b}$ of node $b$ is smaller than $S_{\text {threshold }}$. Because the SGG considers stability, links $b-c$ and $b-e$ will not be built; therefore, the SGG can decrease the energy consumption due to relinking.

The SGG is defined as follows: with an edge $(b-c)$, as shown in Fig. 4, if the link from node $b$ to node $c$ does not include node a and the stability of node $a$ is larger than $S_{\text {threshold }}$, a circle is formed with the diameter of the distance of $b-c$, as in

$$
\forall a \neq b, v:\left\{d^{2}(b, c)<\left[d^{2}(b, a)+d^{2}(c, a)\right]\right\} \cap\left\{S_{a}>S_{\text {threshold }}\right\} .
$$

The SGG routing message is transferred sequentially along the faces with the left-hand and right-hand rules. The SGG considers the node stability and reduces the energy consumption due to relinking by the left-hand and right-hand rules, as shown in Fig. 5. The SGG is combined with planar polygons that are divided into several internal and external faces. The routing message forwarded by the left-hand rule is $\mathrm{S}-\mathrm{V}_{5}-\mathrm{V}_{4}-\mathrm{V}_{3}-\mathrm{V}_{2}-\mathrm{V}_{1}-\mathrm{S}$ and that by the right-hand rule is $\mathrm{S}-\mathrm{V}_{1}-\mathrm{V}_{2}-\mathrm{V}_{3}-\mathrm{V}_{4}-\mathrm{V}_{5}-\mathrm{S}$. The $\mathrm{S}$-mode addresses the dead-end node in the local maximum problem via the SGG.

\section{Validation Result}

In this section, we compare the performance of the proposed SDRFLC methods with that of the SDR. We choose the field area of $4500 \times 2500 \mathrm{~m}^{2}$. The numbers of nodes are $150,250,350,450$, and 550. The duration of simulation is $1000 \mathrm{~s}$. The speed of node mobility ranges from 0 to $10 \mathrm{~m} / \mathrm{s}$. The packet size is 512 bytes. The communication type is CBR(UDP). The mobility model is the random walk model (RWM). The radio range is $100 \mathrm{~m}$. The performance evaluation is divided into two types: delivery ratio and routing overhead. The delivery ratio refers to a node's packet success rate. At the time of transmission, each node passes data through a forwarding node, and the packet loss rate is 0.001. Routing overhead is the energy consumption of a node broadcasting a 32 bit Hello message to find a forwarding node. The simulation is written in $\mathrm{C}$.

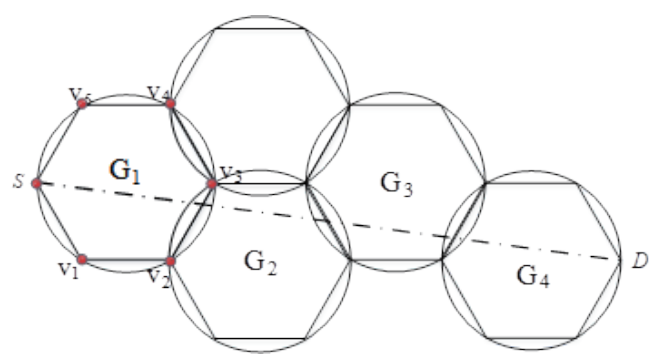

Fig. 5. (Color online) SGG of routing message. 


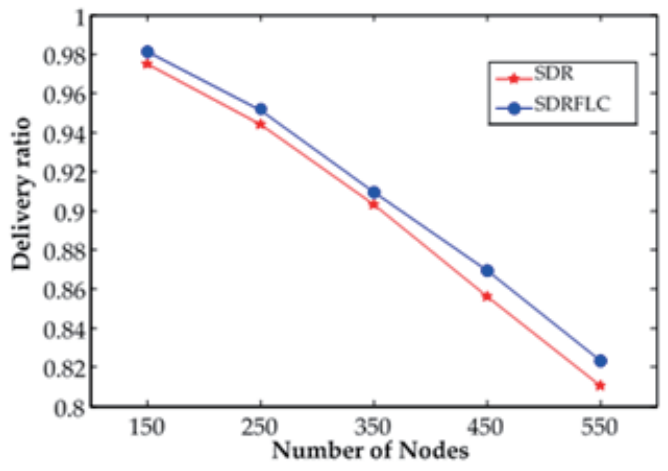

Fig. 6. (Color online) Number of nodes vs delivery ratio.

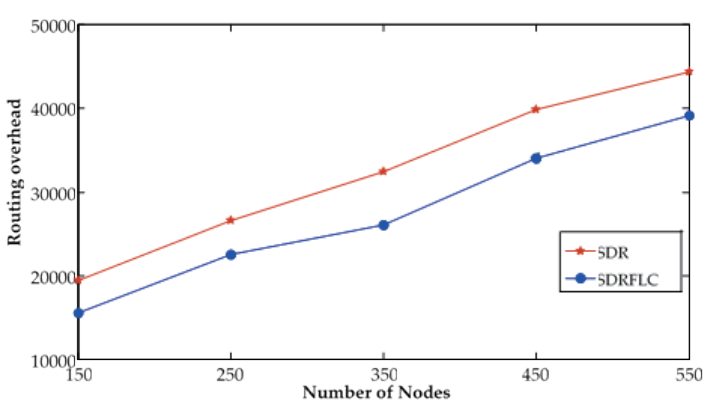

Fig. 7. (Color online) Number of nodes vs routing overhead.

To further compare the performance of the proposed methods with that of the SDR, we consider the delivery ratio of the number of nodes in the field, as shown in Fig. 6. The proposed SDRFLC methods yield better performance results than does the SDR method. The delivery ratio of the SDRFLC ranges from 0.6 to $1.5 \%$ higher than that of the SDR. Furthermore, the routing overhead of the number of nodes in the field is shown in Fig. 7. The proposed SDRFLC methods yield higher performance characteristics than does the SDR method. The routing overhead performance of the SDRFLC, which ranges from 12 to $20 \%$, is also higher than that of the SDR. These performance characteristics are improved because the SDRFLC method improves the DFA facing angle from 180 degrees to an optimum value, which generates a new search angle by fuzzy logic control.

\section{Conclusions}

We proposed the use of an optimum DFA facing angle with the newest S-mode to improve the SDR routing protocol by fuzzy logic control. A corresponding routing mode is selected in accordance with the density value. When the node density is higher than the threshold, the SDRFLC methods will use the original D-mode to forward data. If the node density is sparse, the SDRFLC uses the new $\mathrm{S}^{+}$-mode to find forwarding nodes. The $\mathrm{S}^{+}$-mode utilizes fuzzy logic control to enlarge the searching angle. If the searching angle still cannot find the forwarding node, the node will use the stability-based face routing mechanism to find the forwarding node. In the simulation results of the delivery ratio, the proposed SDRFLC method is better than the SDR by 0.6 to $1.5 \%$. In terms of field size and routing overhead, the proposed SDRFLC method is better than SDR by 12 to $20 \%$. The proposed SDRFLC methods are thus able to reduce routing overhead and extend the mobile ad hoc network lifespan.

\section{Acknowledgments}

This work was supported by the Ministry of Science and Technology (MOST) of the Republic of China under grant Nos. MOST 107-2221-E-025-009 and MOST 105-2221-E-025006. 


\section{References}

1 S. Chatterjee and S. Das: Inf. Sci. 295 (2015) 67. https://doi.org/10.1016/j.ins.2014.09.039

2 A. Moussaoui and A. Boukeream: J. Network Comput. Appl. 47 (2015) 1. https://doi.org/10.1016/ j.jnca.2014.09.007

3 L. Venkatraman and D. P. Agrawal: J. Parallel Distrib. Comput. 63 (2003) 214. https://doi.org/10.1016/S07437315(02)00065-5

4 R. Ye, A. Boukerche, H. Wang, X. Zhou, and B. Yan: Comput. Networks 110 (2016) 351. https://doi. org/10.1016/j.comnet.2016.10.010

5 T. T. Son, H. L. Minh, G. Sexton, and N. Aslam: Ad Hoc Networks 14 (2014) 2. https://doi.org/10.1016/ j.adhoc.2013.10.012

6 E. K. Asl, A. Noorhosseini, and A. S. Pirouz: J. Inf. Sci. Eng. 27 (2011) 1581. https://pdfs.semanticscholar.org/ a18b/5506d9316dd785910cce698bd38a2db77348.pdf

7 M. Saleem, I. Ullah, and M. Farooq: Inf. Sci. 200 (2012) 38. https://doi.org/10.1016/j.ins.2012.02.024

8 V. V. Mandhare, V. R. Thool, and R. R. Manthalkar: Comput. Networks 110 (2016) 180. https://doi.org/10.1016/ j.comnet.2016.09.023

9 A. Moussaoui, F. Semchedine, and A. Boukerram: J. Network Comput. Appl. 39 (2014) 117. https://doi. org/10.1016/j.jnca.2013.05.014

10 P. Jacquet, P. Muhlethaler, T. Clausen, A. Laouiti, A. Qayyum, and L. Viennot: Proc. IEEE Int. Multi Topic Conf. (IEEE, 2001). https://doi.org/10.1109/INMIC.2001.995315

11 Z. J. Haas: Proc. 6th Int. Conf. on Universal Personal Communications (IEEE, 1997). https://doi.org/10.1109/ ICUPC.1997.627227

12 B. Karp and H.T. Kung: Proc. 6th Annual Int. Conf. Mobile Computing and Networking (ACM, 2000). https:// doi.org/10.1145/345910.345953

13 S. K. Arora and H. Monga: Optik - Int. J. Light. Electron. Opt. 127 (2016) 7283. https://doi.org/10.1016/ j.ijleo.2016.04.138

14 I. A. Umar, Z. M. Hanapi, A. Sali, and Z. A. Zulkarnain: Sensors 16 (2016) 943. https://doi.org/10.3390/ s16060943

15 W. Liu and W. Kim: J. Syst. Archit. 59 (2013) 767. https://doi.org/10.1016/j.sysarc.2013.05.004

16 Y. L. Chen and H. P. Lai: Appl. Soft Comput. 14 (2014) 594. https://doi.org/10.1016/j.asoc.2013.08.001

17 Y. L. Chen, J. S. Sheu, and W. X. Huang: Sens. Mater. 27 (2015) 675. https:/doi.org/10.18494/SAM.2015.1221

18 W. B Heinzelman, A. P. Chandrakasan, and H. Balakrishnan: IEEE Trans. Wireless Commun. 1 (2002) 660. https://doi.org/10.1109/TWC.2002.804190

\section{About the Authors}

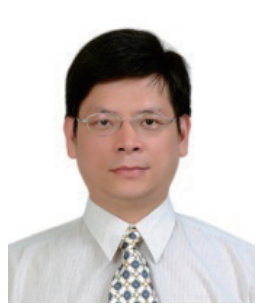

Young-Long Chen received his B.S. degree in automatic control engineering from Feng Chia University, Tai-Chung, Taiwan, in 1988, M.S. degree in engineering science from National Cheng Kung University, Tainan, Taiwan, in 1995, and Ph.D. degree in electrical engineering from National Chung Cheng University, Chia-Yi, Taiwan, in 2007. From 1995 to 1999, he worked for Formosa Petrochemical Corporation as a design engineer. From 1999 to 2007, he was a lecturer with the Department of Electrical Engineering, Chienkuo Technology University, Taiwan. From 2007 to 2009, he was an associate professor with the Department of Electrical Engineering, Chienkuo Technology University, Taiwan. Since 2009, he has been with the Department of Computer Science and Information Engineering, National Taichung University of Science and Technology, Taiwan, where he is currently a professor. His research interests include wireless and mobile communications and networks, wireless sensor networks, information security, digital signal processing, fuzzy neural networks, and embedded systems. He is a member of the IEEE. (ylchen66@nutc.edu.tw) 


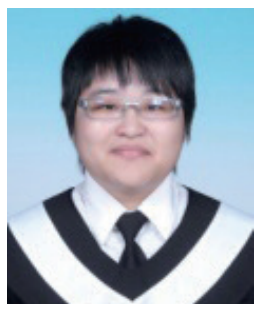

Yu-Ling Zeng received her B.S. degree from the Department of Computer Science and Information Engineering of National Quemoy University, Kinmen, Taiwan, in 2013, and her M.S. degree from the Department of Computer Science and Information Engineering, National Taichung University of Science and Technology, Taichung, Taiwan, in 2015. Her research interests include embedded systems, mobile ad hoc networks, and wireless sensor networks. (blue_sky826@hotmail.com)

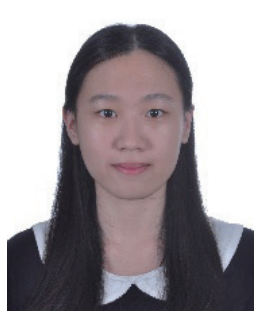

Hsin-Yu Chen received her B.S. degree from the Department of Computer Science and Information Engineering, National Taichung University of Science and Technology, Taichung, Taiwan, in 2017. Since 2017, she has been working toward her M.S. degree. Her research interests include embedded systems, wireless sensor networks, and sensors. (s1810632004@gms.nutc.edu.tw)

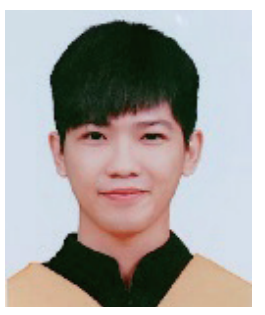

Yan-Hao Lin received his B.S.degree from the Department of Information and Communication Engineering, Chaoyang University of Technology, Taichung, Taiwan, in 2015 and his M.S. degree from the Department of Computer Science and Information Engineering, National Taichung University of Science and Technology, Taichung, Taiwan, in 2017. His research interests include embedded systems, wireless communication networks, and wireless sensor networks. (s10030027@gmail.com) 